
March 1939

\title{
A PRECISION RADIO INSTRUMENT FOR TRANSMITTING MEASUREMENTS OF ULTRAVIOLET INTENSITIES FROM UNMANNED BALLOONS TO A GROUND STATION
}

\author{
By R. Stair
}

\section{ABSTRACT}

A new radio stratosphere ultraviolet meter which operates with a precision comparable to that of laboratory instruments is described. It consists of a photoelectric cell, a balanced amplifier, a relaxation oscillator, and a transmitter. The calibration of the instrument is automatically determined and broadcast to the receiving station each time an altitude signal is given. The instrument, with accessories, including sufficient batteries to operate for about 4 hours, weighs about 5 pounds. It operates on a frequency of approximately 50 megacycles and has sufficient power to insure good reception for distances from the receiving station up to 100 miles.

The present instrument was developed subsequent to the making of six flights into the stratosphere with a three-tube unit during the summer of $1937[1],{ }^{1}$ although a laboratory model was tested and briefly mentioned in the report covering that work.

Because of the very high photoelectric cell and grid resistances involved, the three-tube instrument was difficult to either operate or calibrate; in that it was subject to instability as the result of the relaxation oscillator being worked beyond its stable range. Furthermore, changes in sensitivity and proportionality of response, as the battery voltages dropped, offered insurmountable difficulties in the use of that instrument.

The present instrument, shown diagrammatically in figure 1, virtually combines the balanced direct-current amplifier [2] previously designed for precision work in the laboratory with a relaxation oscillator and short-wave transmitter [3] similar to that developed by Diamond and his collaborators. Sufficient power and sensitivity are available in the new instrument for the relaxation oscillator to operate under normal conditions of circuit and voltage and hence in a condition of stable equilibrium.

Briefly described, the balanced direct-current amplifier, built in the form of a Wheatstone bridge, becomes unbalanced when light falls upon the photoelectric cell. Here the effect produced in the plate circuit, which is also the grid circuit of the relaxation oscillator tube $1 A 6$, may be made several hundred times that possible when using the photoelectric cell coupled directly to the relaxation oscillator, as in the earlier instrument. Thus, in the new instrument, the resistance in the grid circuit of this tube is reduced from hundreds to a few megohms (to 2.6 megohms in the instrument as described). With a

\footnotetext{
${ }^{1}$ Figures in brackets indicate the literature references at the end of this paper.
} 


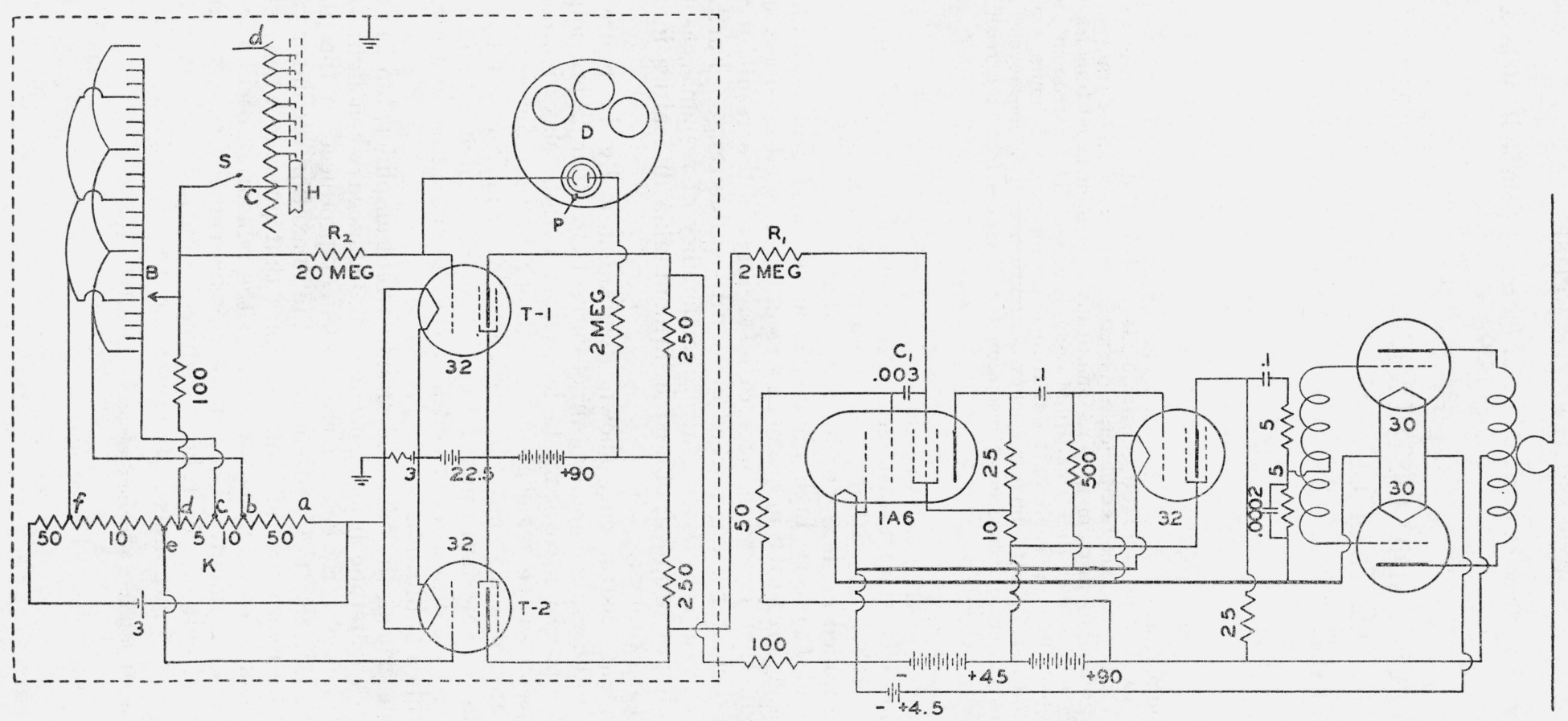

FIGURE 1.-Electrical circuit of the new stratosphere ultraviolet meter.

Resistances are given in thousands of ohms and capacitances in microfarads, except where definitely specified otherwise. 
grid resistor of this magnitude, the relaxation oscillator operates normally and thus repeats consistently from day to day.

The capacitance, $C_{1}$, and the resistance, $R_{1}$, plus the additional resistors between $C_{1}$ and grid 1 of the relaxation oscillator tube $1 \mathrm{~A} 6$, determine the sensitivity and the base value (oscillation frequency with no light on the photoelectric cell) of the relaxation oscillation. Values of $0.003 \mu \mathrm{f}$ and 2.6 megohms, respectively, used in this instrument, give a base frequency of about 50 cycles and provide the correct sensitivity for coupling with the balanced amplifier as used here, when the other constants of the $1 A 6$ tube circuit are as given in figure 1.

The generated audio-frequency voltage is next amplified and finally modulates a push-pull radio-frequency oscillator coupled to a halfwave doublet antenna. The grid and plate coils are closely coupled, being alternately wound with No. 16 enameled copper wire on a $7 / 8$-in. Pyrex tube.

Each coil consists of six turns of wire, center tapped. The antenna coupling coil consists of one turn of wire near the center of the oscillator coils. The antenna is adjusted for maximum power output of the oscillator which operates at approximately $50 \mathrm{Mc}(6 \mathrm{~m})$. Tests have sbown that the transmitter has sufficient power to insure staticfree reception and hence a good record up to a distance of 100 miles from the recording station.

Having the relaxation oscllator and transmitter properly operating, the direct-current amplifier is next adjusted. The two type 32 tubes must be closely similar in characteristics or they will not work satisfactorily in this circuit.

The bias on tube $T-2$, is adjusted to approximately $-1.5 \mathrm{v}$ (point, $e$, on the potentiometer, $K$ ). Next, the connection, $d$, is adjusted to give the proper bias on tube, $T-1$, so that but little change is observed in the relaxation oscillation frequency when the amplifier is turned on; a slight increase in frequency is to be preferred to that of a decrease.

The value of the grid resistor, $R_{2}$, is so chosen as to give the proper sensitivity required of the amplifier for use with the particular photoelectric cell, $P$, employed in the unit. A change in $R_{2}$ ordinarily calls for a change in the bias of $T-1$, since the accompanying grid-current change is sufficient to unbalance the amplifier and hence modify the relaxation oscillation frequency.

In operation, as a small motor [1] rotates the disk, $D$, carrying various filters over the photoelectric cell, the amplifier becomes unbalanced in a degree proportional to the intensity of light reaching the cell. This unbalance in the plate circuit of the amplifier gives rise to a new voltage difference in the grid circuit of the $1 A 6$ relaxation oscillator tube. This voltage change causes in turn an increase in the control grid current in the tube, thus increasing the charging rate of condenser, $C_{1}$, and hence the frequency of oscillation. Tests using perforated screens of known transmission in the path of light show a closely linear change in audio-frequency oscillation with light intensity. The several instruments built and tested gave frequency changes which were linear to within about 1 percent throughout the range of 50 to 200 $\mathrm{c} / \mathrm{s}$, for which they were designed. Figure 2 shows a record when three perforated screens having transmissions of $12.9,48.1$, and 91 percent were being used to test the performance of the instrument. As a further precaution, in practice, each optical filter is used with an 
accompanying perforated screen having approximately the same transmission so that any possible deviations from linearity in either the radiometeorograph or the ground-station receiver and recorder are automatically compensated. This is very important since the calculations on the spectral quality of the ultraviolet radiation and the amount of ozone penetrated are based upon the absolute value of, and changes in, the filter transmissions as the instrument reaches higher altitudes.

The barograph, $B$, not only indicates the altitude as the arm makes contact with a selected segment, $b, c$, or $f$, but impresses a definite voltage, for example, that across $c d$, on the grid of tube, $T-1$, which in turn causes an increase in audio-frequency oscillation indicative of the absolute sensitivity of the complete instrument. This is illustrated by the change in frequency of the base line from $d$ to $c$ in figure 2 . When a contact is made with a segment connected to $b$ the base

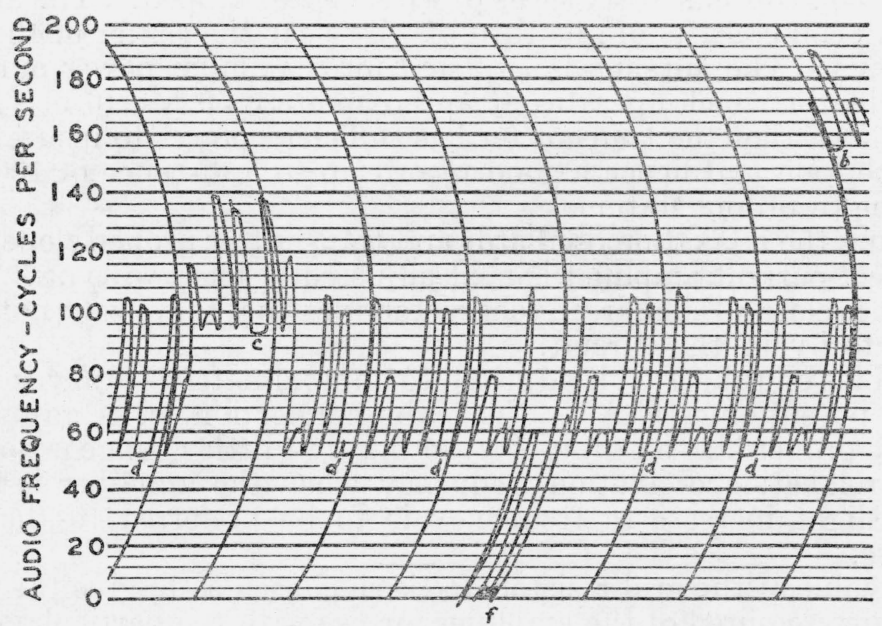

FIGURE 2.-Chart showing type of record transmitted.

frequency is raised still higher. On the other hand, when a contact is made to $f$ the base frequency is lowered, and may be made zero. In practice, the contacts $b$ and $f$ are used as reference points of altitude only, while the more numerous contacts, $c$, are used both for sensitivity calibration and altitude determination.

A projected modification of the barograph is the addition of a small auxiliary mercury $U$-tube unit to give an absolute indication of pressure, and hence, altitude for elevations above $60,000 \mathrm{ft}(18 \mathrm{~km})$. This modification will also give an automatic sensitivity test for each cycle of measurements, since the switch, $S$, is to be closed once during each rotation by a cam on the filter disk shaft. This unit is to be placed in the potentiometer circuit, $K$, between $c$ and $d$. (See upper part of fig. 1.) That is, the resistor, $c d$, is to be broken, say into $1,000-\mathrm{ohm}$, steps, each being connected to an electrode in the small mercury U-tube, so that as the pressure decreases and the mercury rises in the open end of the tube, successive sections of the resistor are short-circuited, resulting in decreased audio-frequency changes upon the closing of switch, $S$. These changes will be easily recognizable on the record. 
As shown in figure 1, the direct-current amplifier, barograph, $B$, photoelectric cell, $P$, and all connecting leads are completely shielded with aluminum foil which is grounded to the negative of the filament battery. This is necessary in order to eliminate electrostatic effects which would otherwise be uncontrollable in the calibration and use of the instrument.

The relaxation oscillator and transmitter require a separate set of filament and plate batteries since they operate at a potential which constantly varies relative to that of the amplifier. Furthermore, it is necessary that the two units be carefully insulated or variable leakages will produce inconstancy in the base of the audio-frequency signal. For this purpose the transmitter is mounted on Bakelite and the transmitter batteries are wrapped in thin rubber.

The aim has been to make the various units of this instrument small and light in weight and yet retain materials of sufficiently good quality to insure precision operation. All tube bases are removed and the wires soldered directly to the protruding electrodes. The batteries have been chosen of minimum size sufficient to give about 3 to 4 hours of dependable operation at the reduced temperatures encountered. For the filament current in the balanced amplifier, two standard flashlight cells in series are used to operate the two type 32 tubes in parallel. The four tubes in the relaxation oscillator transmitter unit are wired in series parallel, so that three standard flashlight cells in series give the same useful life as the two in the amplifier. Batteries of the Burgess types VBOFL and WBOFL are employed for the plate voltages in the amplifier and transmitter, respectively.

For the potentiometer, $K$, two type $Z$ Penlite cells in series are permanently shunted by approximately $120,000 \mathrm{ohms}$. After a few days, they reach an equilibrium value which changes but little over a period of weeks. Upon the constancy of these cells and resistors depends the calibration of the radiometeorograph from the time it is tested in the laboratory until the flight is finished.

For lightness and compactness most of the resistor units are of the standard 0.5-watt type. In the grid and plate circuits of the balanced amplifier, however, S. S. White or wire-wound units are used to give more reliable and stable operation of the amplifier. This precaution is very important since a small change in this part of the circuit, for example, a defective resistor in the plate circuit of one of the amplifier tubes, may, through fluctuation in magnitude, shift the audio-frequency base level ( $d$, in fig. 2 ) off the scale in either the positive or negative direction. A slight drift, usually downward, generally occurs as the battery voltages decrease. This is not a serious matter, however, as long as the drift is not sufficient to reach the nonlinear portion of the scale toward the lower frequencies, because the absolute sensitivity of the complete instrument (amplifier and relaxation oscillator) is checked each time a barograph contact is made.

The complete instrument (weighing about $5 \mathrm{lb}$.) compactly inclosed in a small balsa-wood box ( 6 by 6 by 9 in.) is wrapped in cotton and black cloth to prevent the batteries from chilling at the low temperatures prevailing in the stratosphere. The box is supported at the four corners by linen threads adjusted to keep the photoelectric cell horizontal, and attached to the upper half of the antenna which in 
turn is used for supporting the instrument and incidentally kept taut. The lower half of the antenna is kept stretched by a small weight attached to the lower end. Thus the radio frequency is held fairly constant and fading because of frequency shift is minimized.

Details on the characteristics of the cadmium-alloy photoelectric cell, the optical filters used, and a discussion of the method of evaluating the data for determining the intensity of the ultraviolet radiation and the distribution of ozone in the stratosphere have been outlined in the report on the 1937 flights [1]. Data obtained in the six flights with this instrument during June 1938 are being assembled and will be published in the Journal of Research of the National Bureau of Standards [4].

Although the instrument as described here was designed for use in the study of ultraviolet intensities and ozone distribution in the stratosphere while being transported by small unmanned balloons, its usefulness may be extended to other fields. The method of coupling a receiver of low sensitivity (the photoelectric cell) with a relaxation oscillator offers a practical means for recording directly, or for transmitting to a remote station before recording, any data obtainable in the form of variation in radiation intensity. For a stationary instrument a more elaborate unit with greater flexibility of adjustment and control would be desired. In case the radio transmitter is omitted, the audio-frequency signal from the relaxation oscillator, after proper amplification, would be impressed directly upon the grid circuit of the frequency meter in the recording system.

[1] R. Stair and W. W. Coblentz, J. Research NBS 20, 185 (1938) RP1075.

[2] W. W. Coblentz and R. Stair, J. Research NBS 12, 231 (1934) RP647.

[3] H. Diamond, W. S. Hinman, Jr., and F. W. Dunmore, J. Research NBS 20, 369 (1938) RP1082.

[4] W. W. Coblentz and R. Stair, J. Research NBS 22 (May 1939).

Washington, December 20, 1938. 